
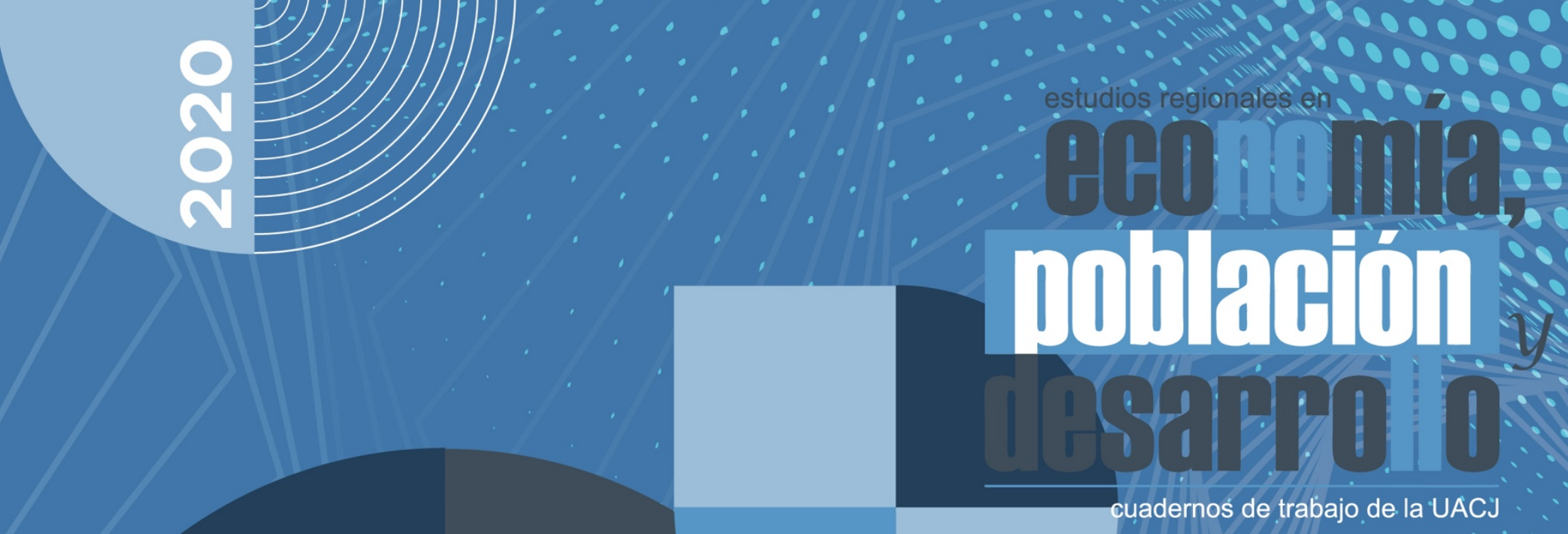

\title{
El índice de desarrollo de TIC en las economías urbana y rural de México
}

Marlen Martínez Domínguez y

Jimena Méndez Navarro

$\therefore:$

$\ldots$

$\therefore \ldots$

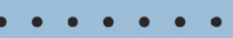

$\bullet \bullet \bullet \bullet \bullet \bullet \bullet \bullet \bullet \bullet \bullet \bullet, \bullet$

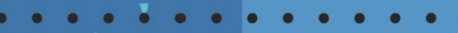

$\bullet \bullet \bullet \bullet \bullet \cdot \bullet \cdot \bullet \bullet \bullet \bullet \bullet \bullet$

$\bullet \bullet \bullet \bullet \bullet, \bullet \bullet \bullet \bullet \bullet$

, $\bullet \bullet \bullet, \bullet \cdot \bullet \cdot \bullet$

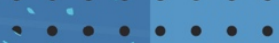

9 


\section{El índice de desarrollo de TIC en las economías urbana y rural de México}

Marlen Martínez Domínguez y Jimena Méndez Navarro

\section{UNIVERSIDAD AUTÓNOMA DE CIUDAD JUÁREZ \\ PUBLICACIÓN AFILIADA A LA \\ RED IBEROAMERICANA DE ESTUDIOS DEL DESARROLLO}




\title{
UNIVERSIDAD AUTÓNOMA DE CIUDAD JUÁREZ
}

\author{
PUBLICACIÓN AFILIADA A LA \\ RED IBEROAMERICANA DE ESTUDIOS DEL DESARROLLO
}

\section{Universidad Autónoma de Ciudad Juárez}

2018-2024

Mtro. Juan Ignacio Camargo Nassar

Rector

Mtro. Daniel Alberto Constandse Cortez

Secretario General

Mtro. Santos Alonso Morales Muñoz

Director del Instituto de Ciencias Sociales y Administración

Mtro. Jesús Meza Vega

Director General de Comunicación Universitaria

Comité de Coordinación de la Red Iberoamericana

de Estudios del Desarrollo 2018-2020

Dra. Paulina Sanhueza Martínez (Universidad de la Frontera, Chile)

Coordinadora General

Dr. Ignacio Rodríguez Rodríguez (Universidad de la Frontera, Chile)

Secretario general

Dra. Myrna Limas Hernández

(Universidad Autónoma de Ciudad Juárez, México)

Vocal de Organización

Dr. Pablo Galaso Reca (Universidad de la República, Uruguay)

Vocal de Organización

Dr. Luis Enrique Gutiérrez Casas

Director y editor de Cuadernos de Trabajo

Estudios Regionales en Economía, Población y Desarrollo

Comité editorial

Sección internacional

Dra. Sofía Boza Martínez

(Universidad de Chile, Chile)

Dra. Olga Biosca Artiñano

(Glasgow Caledonian University, Reino Unido)

Dra. Ángeles Sánchez Díez

(Universidad Autónoma de Madrid, España)

Dr. Thomas Fullerton Mankin

(University of Texas at E1 Paso, Estados Unidos)

Dr. Adrián Rodríguez Miranda

(Universidad de la República, Uruguay)

Dra. Ikuho Kochi

(Kanazawa University, Japón)

Sección local

(Universidad Autónoma de Ciudad Juárez)

Dra. Myrna Limas Hernández

Dra. Rosa María García Almada

Dr. Raúl Alberto Ponce Rodríguez

Dr. Isaac Leobardo Sánchez Juárez

Dr. Héctor Alonso Barajas Bustillos

Dr. Juan Carlos Medina Guirado

Diseño de cubierta Abigail Bautista
Estudios Regionales en Economía, Población

y Desarrollo. Cuadernos de Trabajo de la UACJ

ISSN 2007-3739

Número 60. Noviembre - Diciembre 2020

El índice de desarrollo de TIC en las economías

urbana y rural de México

Marlen Martínez Domínguez y Jimena Méndez Navarro

Universidad Autónoma de Ciudad Juárez

Estudios Regionales en Economía, Población y Desarrollo.

Cuadernos de Trabajo de la UACJ

Año 10, No. 60 noviembre - diciembre 2020, es una publicación bimestral editada por la Universidad Autónoma de Ciudad Juárez a través del Cuerpo Académico de Estudios Regionales en Economía, Población y Desarrollo del Instituto de Ciencias Sociales y Administración. Redacción: Avenida Universidad y H. Colegio Militar, Zona Chamizal s/n., C.P. 32300, Ciudad Juárez, Chihuahua, México.

Teléfonos: (656) 688-38-00, ext.3792. Correo electrónico: igtz@uacj.mx. Editor responsable: Luis Enrique Gutiérrez Casas. Reserva de derechos al uso exclusivo: edición impresa, ISSN 2007-3739., edición digital, No. de reserva 04-2019-050218151500. Impresa por Studio Los Dorados, calle Del Campanario, número 820-2, Santa Cecilia, C.P. 32350, Cd. Juárez, Chihuahua. Distribuidor: Subdirección de Gestión de Proyecto y Marketing Editorial. Ave. Plutarco Elías Calles 1210, Foviste Chamizal, C.P. 32310, Ciudad Juárez, Chihuahua. Este número se terminó de imprimir el 15 de octubre 2020 con un tiraje de 120 ejempares.

Los ensayos publicados son responsabilidad exclusiva de sus autores.

Se autoriza la reproducción total o parcial bajo condición de citar la fuente.

\section{Registrada en: EBSCO RePFC}

Publicación afiliada a la Red Iberoamericana de Estudios del Desarrollo

Universidad Autónoma de Ciudad Juárez

Ave Plutarco Elías Calles 1210

Foviste Chamizal, C.P. 32310

Ciudad Juárez, Chihuahua, México

www.uacj.mx

(C) Universidad Autónoma de Ciudad Juárez 


\title{
El índice de desarrollo de TIC en las economías urbana y rural de México
}

\author{
Marlen Martínez Domínguez * y Jimena Méndez Navarro **
}

\begin{abstract}
Resumen
El objetivo del artículo es medir la brecha digital en zonas urbanas y rurales por entidad federativa en México, utilizando la metodología del índice de desarrollo de las TIC de la Unión Internacional de Telecomunicaciones, con información de la Encuesta Nacional sobre Disponibilidad y Uso de Tecnologías de la Información en los Hogares (ENDUTIH) 20172018. Los resultados muestran mayor desarrollo de las TIC, en particular en las zonas urbanas de los estados del sur y norte del país. Asimismo, los hallazgos reflejan que existe asociación entre los niveles altos de acceso, uso y capacidades de las TIC y la digitalización del sector terciario en la economía.
\end{abstract}

Palabras clave: TIC, acceso, uso, apropiación, rural, urbano.

\begin{abstract}
The objective of the article is to measure the digital gap in urban and rural areas in Mexico by state, using the methodology of the ICT development index of the International Telecommunication Union, with information from the National Survey on Availability and Use of Information Technologies in Households (ENDUTIH) 2017-2018. The results show further development of ICT, particularly in urban areas of the southern and northern states of the country. Likewise, the findings reflect that there is an association between high levels of ICT access, use and capabilities and the digitization of the tertiary sector in the economy. Keywords: ICT, access, use, appropriation, rural, urban.
\end{abstract}

JEL: 018, 033, R19.

Recibido en: julio de 2020.

- Aprobado en: octubre de 2020.

* Cátedra Conacyt en Centro de Investigaciones y Estudios Superiores en Antropología Social (CIESASPacífico Sur). Líneas de investigación: desarrollo económico, apropiación de TIC y pobreza. Correo electrónico: mmartinez@ciesas.edu.mx.

** Colaboradora independiente. Líneas de investigación género, migración y desarrollo rural. Correo electrónico: mendedezjimena33@gmail.com. 


\section{$\rightarrow$ 1. Introducción.}

Las Tecnologías de la Información y Comunicación (TIC) son presentadas como factores para el desarrollo socioeconómico global, debido a que pueden desempeñar un papel significativo en el desarrollo económico, el crecimiento del Producto Interno Bruto (PIB), el empleo, la productividad, la reducción de la pobreza e incentivar la participación democrática de los ciudadanos, de acuerdo con Goncalves (2018) y Palvia (2018).

La información es un elemento indispensable en la toma de decisiones de las estrategias de sustento de los hogares, de esta manera, las TIC representan oportunidades porque reducen el tiempo y costo de acceso a los mercados; mayor y mejor información conlleva a una toma de decisiones de consumo y producción óptimas; eficiencia y productividad en las actividades económicas; mayor expansión del mercado; empoderamiento de las comunidades rurales; acceso a la educación a distancia y la generación de empleos según Felizzola (2010), Torero y Von Braun (2006).

La brecha digital es una expresión de las desigualdades en el contexto del desarrollo de la sociedad de la información y el conocimiento (Martínez-Domínguez, 2018; De la Selva, 2015; Van Dijk, 2006). De acuerdo con la OCDE (2001:5), se define como "la brecha entre individuos, hogares, negocios y áreas geográficas en diferentes niveles socioeconómicos con respecto a sus oportunidades de acceso a TIC y su uso para una amplia variedad de actividades".

A pesar de que la digitalización de la sociedad está aumentando en todo el mundo, la brecha digital sigue persistiendo y representa un relevante factor de exclusión y marginación a nivel global (Szeles, 2018; Goncalves, 2018; Tirado-Morueta, 2017; Nishijima,, 2017; Malecki, 2003). De acuerdo con información de la Unión Internacional de Telecomunicaciones (ITU por sus siglas en inglés), México ocupa los últimos lugares de los países de América Latina en la medición de la sociedad de la información a escala mundial (ITU, 2018).

El presente artículo tiene como objetivo medir la brecha digital a escala urbana y rural por entidad federativa en México, utilizando la metodología de la ITU y analizar la relación entre brecha digital y condiciones estructurales del empleo por sectores económicos. La contribución del estudio es analizar la brecha digital a partir de la construcción del índice de desarrollo de TIC a escala urbana y rural por entidad con el propósito de aportar indicadores que permitan a los hacedores de política pública diseñar medidas efectivas, para avanzar en la digitalización como un tema prioritario para alcanzar el crecimiento económico y la reducción de la pobreza (Szeles, 2018).

La relación entre índice de apropiación tecnológica y desarrollo socioeconómico se vincula con la noción de brecha digital que surge en los Estados Unidos en la década de los noventa con Gunkel (2003). Esta consta de tres categorías de análisis. La primera se refiere al acceso, en un inicio 
lo relacionaron con las desigualdades en infraestructura y servicios (Van Dijk, 2006; Selwyn, 2004); sin embargo, estudios recientes de Szeles (2018) y Scheerder (2017) mostraron que el acceso está influido por factores socioeconómicos como el ingreso, la ubicación geográfica, el nivel educativo, la edad y las habilidades digitales. Los estudios de este nivel mostraron que el acceso a las TIC se distribuye de manera desigual entre las personas con diferentes características demográficas como edad, género, nivel socioeconómico, origen étnico y geografía (Helsper, 2010; Mossberger, 2003). La segunda fase es el uso, donde las habilidades y aplicaciones son explicadas por diferencias sociales (Dewan y Riggins, 2005; Van Dijk y Hacker, 2003). Las investigaciones efectuadas sobre esta etapa han explorado el tipo de actividades que las personas realizan en línea, así como las habilidades requeridas para este propósito (Van Deursen, 2017; Van Deursen y Van Dijk, 2014). Por último, se encuentran los beneficios, resultado del uso de las TIC (Van Deursen y Helsper, 2015; Blank y Groselj, 2014). Los trabajos de Van Deursen (2017) y Scheerder (2017) han examinado a aquellos individuos que se benefician del uso de internet

La digitalización es un tema ampliamente abordado en los países desarrollados y en desarrollo. En el caso de México, existen pocos estudios centrados en las TIC (Márquez., 2016; Toudert, 2013). Al respecto, Mariscal (2005) muestra que la desigual distribución regional en teledensidad genera una brecha digital mayor. Asimismo, Martínez-Domínguez (2018) demuestra que factores como la edad, el nivel educativo, el ingreso, la ocupación, las habilidades digitales y el lugar de residencia influyen en el acceso y uso de las TIC (computadora, internet y telefonía móvil).

El documento se estructura de la siguiente forma en el desarrollo se presenta el panorama de las TIC en los contextos rurales, se describen los datos y la metodología empleada en la construcción del IDT y los resultados. Por último, se presentan las conclusiones y las reflexiones finales.

\section{2. Panorama de las TIC en el contexto rural y urbano.}

A pesar de los avances en los años recientes, la brecha digital en zonas urbanas y rurales persiste entre los países miembros y asociados de la Organización para la Cooperación y el Desarrollo Económico (OCDE), por ejemplo la disponibilidad y conexión a internet en hogares rurales fue menor que en hogares urbanos, en particular, en países como México, Chile y Colombia de acuerdo con la OCDE (2018).

Con respecto a la conectividad digital en las áreas rurales, Salemink (2017) y Townsend (2013) encuentran la presencia de escasos proveedores, altos costos, una difusión más lenta del internet y una limitada infraestructura. Esto se explica porque para las empresas de telecomunicaciones privadas 
les genera poca rentabilidad proporcionar el servicio en las zonas rurales, debido a las bajas densidades de población y a su aislamiento Whitacre y Mills (2007) y Malecki (2003). No obstante, existe evidencia que la infraestructura digital puede desempeñar un papel clave en el fortalecimiento del desarrollo económico de las comunidades rurales de forma indirecta, al permitir el comercio en línea, expandir los mercados, obtener información sobre el mercado y los productos, según Gwaka (2018) y Aker (2016).

A pesar de que el acceso a internet ha mejorado en las zonas rurales de los países desarrollados, se observa que la adopción no se traduce en automático en mayor uso, lo que sugiere que la brecha digital rural se encuentra arraigada a aspectos socioeconómicos, demográficos, étnicos y de conocimiento (Hodge, 2017; Salemink, 2017).

Según información de la Encuesta Nacional sobre Disponibilidad y Uso de Tecnologías de la Información en los Hogares (ENDUTIH, 2018), 40.6\% de la población rural de seis años y más es usuaria de internet, en comparación con $73.1 \%$ de las personas que residen en áreas urbanas. Los estados que presentan los más altos valores de usuarios urbanos fueron Sonora, Baja California, Quintana Roo y Nuevo León, en tanto que en las zonas rurales destaca Baja California, Aguascalientes y Morelos. (INEGI, 2018a).

Con base en la ENDUTIH 2017 y 2018 se presenta un conjunto de estadísticas descriptivas que muestran un panorama general de la situación actual del acceso y uso de las TIC en el sector rural y urbano. En las zonas rurales se observa que el uso de internet pasó de 39.2\% a 40.6\%; mientras la telefonía móvil creció de 53.83\% a 55.12\%. Sin embargo, el acceso a la computadora disminuyó de $25.16 \%$ a $24.2 \%$. En el caso de las zonas urbanas, el porcentaje de usuarios de la red aumentó de $71.2 \%$ a 73.1\%; al igual que el uso del celular al pasar de 77.7\% a 78.91\%; asimismo, el acceso a la computadora disminuyó de 51.23\% a 51\% (INEGI, 2017 y 2018a).

De acuerdo con los datos de los años 2017 y 2018, la falta de internet en los hogares urbanos y rurales fue por restricciones económicas. De esos hogares, sus integrantes tienen carencias de habilidades digitales o desconocen su utilidad. Lo anterior hace patente la oportunidad de invertir en la capacitación para la adquisición de habilidades digitales. Por otro lado, los miembros de los hogares de ambos ámbitos señalan al hogar como el principal lugar para usar internet, siendo cualquier otro lugar la segunda opción para conectarse a la red. Cabe señalar que, los usuarios del ciberespacio tanto urbanos como rurales lo usan en mayor medida para el entretenimiento y la comunicación (INEGI, 2017 y 2018a).

En lo que se refiere a políticas de TIC en el país, hasta antes de 2013, el sector de telecomunicaciones estuvo altamente concentrado en una sola empresa (América Móvil y sus dos filiales Telmex y Telcel). En ese periodo hubo bajos niveles de competencia, altas tarifas, baja 
densidad de servicios y débiles agentes de regulación (Ovando y Olivera, 2018; Ayala, 2018). En 2013 el Diario Oficial de la Federación (DOF) promulgó la reforma de telecomunicaciones y radiodifusión, en donde se reconoció el acceso a las TIC como un derecho humano en la Constitución (2013).

En ese mismo año se implementó la Estrategia Digital Nacional, un plan de acciones para fomentar la adopción y desarrollo de las TIC. Esta se basó en cinco objetivos: i) transformación gubernamental; ii) economía digital; iii) educación de calidad; iv) salud universal y efectiva y v) seguridad ciudadana, con cinco habilitadores como la conectividad, la inclusión y las habilidades digitales, la interoperabilidad, el marco jurídico y los datos abiertos (Gobierno de la República, 2013).

\section{○ 3. EI Índice de Desarrollo de las Tecnologías de la Información y Comunicación (TIC).}

Desde el 2009, la ITU publica el informe anual "medición de la sociedad de la información”. Este documento contiene el Índice de Desarrollo de las TIC (IDT) y según resultados de la medición de 2017, México ocupó la posición global 87, precedido por otros países latinoamericanos como Uruguay, Chile, Brasil y Colombia (ITU, 2018). En la medición se señala que el acceso a Internet móvil denota un crecimiento firme y que los usuarios cada vez más utilizan los servicios móviles. Es esencial que el país se oriente hacia una sociedad basada en el conocimiento, donde se reconozca que las TIC son un habilitador del desarrollo si se aplican y usan apropiadamente para el crecimiento y desarrollo económico. Esto ha quedado demostrado con la pandemia del coronavirus, donde las TIC se han convertido en herramientas fundamentales para la educación y la salud. No obstante, además de la existente brecha de acceso a las tecnologías digitales, éstas también se han empleado para difundir noticias falsas, de ahí que es esencial por un lado fomentar el acceso a las TIC y por otro, la alfabetización digital para distinguir la información falsa de la verdadera.

La ITU creó el Índice de Desarrollo de las TIC (IDT), que se compone de 11 indicadores sobre acceso, uso y capacidades de las TIC, el cual permite hacer comparaciones entre países a nivel global. El objetivo del IDT es la medición para comparar y evaluar la evolución de las TIC, la brecha digital y las posibilidades de desarrollo de las TIC entre países (ITU, 2018). El IDT se compone de tres subíndices: acceso, utilización y capacidades; estos elementos corresponden a distintos aspectos del proceso de desarrollo de la apropiación social de las TIC (ver cuadro 1). En este marco internacional de comparación que proporciona el ITU, México se sitúa en el lugar 87 en 2017, con siete economías latinas por delante de él. El IDT fue de 4.91. En el cuadro 2 se presenta una 
clasificación de los primeros 10 países de América Latina, el 2016 y 2017, en el contexto del primer lugar y último lugar del mundo.

Cuadro 1

\section{Componentes, indicadores, ponderación y valores de referencia del Índice de Desarrollo de las TIC}

\begin{tabular}{|c|c|c|}
\hline Acceso & Valor de referencia & $(\%)$ \\
\hline $\begin{array}{l}\text { 1. Suscripciones a telefonía fija por } \\
\text { cada } 100 \text { habitantes }\end{array}$ & 60 & 20 \\
\hline $\begin{array}{l}\text { 2. Suscripciones a telefonía móvil } \\
\text { por cada } 100 \text { habitantes }\end{array}$ & 120 & 20 \\
\hline $\begin{array}{l}\text { 3. ancho de banda de internet } \\
\text { internacional (bits/seg) por usuario } \\
\text { de internet }\end{array}$ & $2^{\prime} 158^{\prime} 212^{*}$ & 20 \\
\hline $\begin{array}{l}\text { 4. Porcentaje de hogares con } \\
\text { computadora }\end{array}$ & 100 & 20 \\
\hline $\begin{array}{l}\text { 5. Porcentaje de hogares con } \\
\text { acceso a internet }\end{array}$ & 100 & 20 \\
\hline Uso de TIC & Valor de referencia & $(\%)$ \\
\hline $\begin{array}{l}\text { 6. Porcentaje de usuarios de } \\
\text { internet }\end{array}$ & 100 & 33 \\
\hline $\begin{array}{l}\text { 7. Suscripciones de banda ancha } \\
\text { fija por cada } 10 \text { habitantes }\end{array}$ & 60 & 33 \\
\hline $\begin{array}{l}\text { 8. Suscripciones de banda ancha } \\
\text { móvil por cada } 10 \text { habitantes }\end{array}$ & 100 & 33 \\
\hline Capacidades para las TIC & Valor de referencia & $(\%)$ \\
\hline 9. Promedio de años de escolaridad & 15 & 33 \\
\hline $\begin{array}{l}\text { 10. Tasa de matrícula de estudios } \\
\text { de nivel secundaria }\end{array}$ & 100 & 33 \\
\hline $\begin{array}{l}\text { 11. Tasa de matrícula de estudios } \\
\text { de nivel terciario }\end{array}$ & 100 & 33 \\
\hline
\end{tabular}

Nota: *Este corresponde al log de 6.33, el cual fue utilizado en la etapa de normalización. Fuente: ITU, 2017. 
Cuadro 2

Lugares de los 10 países de América Latina en la clasificación del Índice de Desarrollo de las TIC

\begin{tabular}{lcccc}
\hline \multicolumn{1}{c}{ País } & $\begin{array}{c}\text { Clasificación } \\
\mathbf{2 0 1 7}\end{array}$ & $\begin{array}{c}\text { Valor del IDT } \\
\mathbf{2 0 1 7}\end{array}$ & $\begin{array}{c}\text { Clasificación } \\
\mathbf{2 0 1 6}\end{array}$ & $\begin{array}{c}\text { Valor del IDT } \\
\mathbf{2 0 1 6}\end{array}$ \\
\hline Islandia & 1 & 8.98 & 2 & 8.78 \\
Uruguay & 42 & 7.16 & 48 & 6.75 \\
Argentina & 51 & 6.79 & 52 & 6.68 \\
Chile & 56 & 6.61 & 59 & 6.28 \\
Costa Rica & 60 & 6.44 & 57 & 6.29 \\
Brasil & 66 & 6.12 & 67 & 5.89 \\
Colombia & 84 & 5.36 & 84 & 5.12 \\
Venezuela & 86 & 5.17 & 82 & 5.22 \\
México & 87 & 5.16 & 90 & 4.87 \\
Panamá & 94 & 4.91 & 93 & 4.8 \\
Perú & 96 & 4.85 & 97 & 4.61 \\
Eritrea & 176 & 0.96 & 175 & 0.96 \\
\hline
\end{tabular}

Fuente: ITU, 2018.

\section{- 4. Metodología para la construcción del IDT en el contexto rural y urbano de México.}

En México, el Instituto Nacional de Estadística y Geografía (INEGI) realiza el levantamiento de la ENDUTIH, la cual tiene como finalidad obtener información sobre la disponibilidad y el uso de las TIC en los hogares y por individuos de seis años y más. A partir de 2015 se aplica la encuesta de forma anual y desde 2017 la información recopilada es representativa del ámbito rural y urbano del país (INEGI, 2018a).

Investigaciones previas de Micheli y Valle (2018) y Ruíz (2015) desarrollan el IDT a nivel estatal, sin hacer diferencia entre zonas rurales y urbanas. La contribución de este estudio es utilizar la información de la ENDUTIH 2017 y 2018 y construir el índice de desarrollo de TIC para el sector rural y urbano de cada una de las 32 entidades federativas.

El cuadro 3 muestra las variables empleadas en la metodología de la ITU y sus pares para la construcción del IDT de las regiones urbanas y rurales de México. Se agrupan en tres subíndices con 
distinto factor de peso en el índice global: a) acceso a las TIC, que identifica la infraestructura para el acceso digital tomando a los hogares como unidades de muestreo, b) el uso, que se refiere a la adopción de internet fijo y/o móvil y c) las capacidades de las personas relacionadas con la educación formal. De forma análoga el IDT urbano y rural de México se conforma de esos tres subíndices y con participación similares al IDT de la ITU.

En la construcción del IDT urbano y rural se consideraron las ponderaciones del IDT de la ITU. Así el factor acceso aporta 40\%; es decir, cuatro de los diez puntos máximos los da la evaluación. A su vez, este factor se integra por cuatro subindicadores con pesos similares (25\%). Los datos para el cálculo provienen de la ENDUTIH 2018, de la sección de hogares.

i. Porcentaje de hogares con telefonía fija, que se deriva de la pregunta 5.4 ¿Dispone de línea de telefónica fija?

ii. Porcentaje de hogares con teléfono móvil, que proviene de la pregunta 4.1.5 ¿Dispone de teléfono celular móvil por cualquiera de los integrantes?

iii. Porcentaje de hogares con computadora o tableta (PC a laptop), a partir de la pregunta 4.2.1., 4.2.2 y 4.2.3 ¿Dispone de computadora de escritorio, computadora portátil o tableta?

iv. Porcentaje de hogares con acceso a Internet, obtenido de la pregunta 4.4 ¿Dispone conexión a internet?

El factor uso aporta 40\% del valor máximo del IDT; sus subfactores son tres y cada uno de ellos tiene un peso similar (33.33\%) en su composición. La información del subfactor 5 proviene de la sección características sociodemográficas, en tanto que 6 y 7 de la sección equipamiento de TIC en el hogar.

v. Porcentaje de población que utiliza Internet, a partir de la respuesta de la pregunta 3.9 ¿En los últimos tres meses ha hecho uso de internet?

vi. Porcentaje de población con conexión alámbrica, procede de la pregunta 4.5 ¿La conexión a internet es fija, móvil o ambas? se considera si tuvo acceso a fija o ambas.

vii. Porcentaje de población con conexión inalámbrica, procede de la pregunta 4.5 ¿La conexión a internet es fija, móvil o ambas? se considera si tuvo acceso a móvil o ambas.

Por último, el factor capacidades aporta $20 \%$ al índice y contiene tres subfactores con pesos similares dentro de él, los cuales se forman a partir de información de los residentes de los hogares de la encuesta. 
viii. Tasa de matrícula en primaria. Porcentaje de población de 6 a 11 años que asiste a la primaria.

ix. Tasa de matrícula en secundaria. Porcentaje de población de 12 a 14 años que asiste a la secundaria.

x. Tasa de matrícula en preparatoria. Porcentaje de población de 15 a17 años que asiste a la preparatoria o similar.

Cuadro 3

Comparativo de las metodologías para el IDT y el IDT Rural y Urbano de México

\section{Metodología ITU para el IDT}

\section{Metodología para el IDT Rural y Urbano de México}

\begin{tabular}{|c|c|c|c|}
\hline Subfactor & $\%$ & Subfactor & $\%$ \\
\hline $\begin{array}{l}\text { 1. Suscripciones a telefonía fija por cada } 100 \\
\text { habitantes }\end{array}$ & $20 \%$ & 1. Porcentaje de hogares con telefonía fija & $25 \%$ \\
\hline $\begin{array}{l}\text { 2. Suscripciones a telefonía móvil por cada } \\
100 \text { habitantes }\end{array}$ & $20 \%$ & $\begin{array}{l}\text { 2. Porcentaje de hogares con acceso a } \\
\text { telefonía móvil }\end{array}$ & $25 \%$ \\
\hline $\begin{array}{l}\text { 3. ancho de banda de internet internacional } \\
\text { (bits/seg) por usuario de internet }\end{array}$ & $20 \%$ & - & \\
\hline 4. Porcentaje de hogares con computadora & $20 \%$ & $\begin{array}{l}\text { 3. Porcentaje de hogares con computadora o } \\
\text { tableta }\end{array}$ & $25 \%$ \\
\hline 5. Porcentaje de hogares con acceso a internet & $20 \%$ & 4. Porcentaje de hogares con acceso a internet & \\
\hline
\end{tabular}

Factor: uso de TIC (40\% en el índice)

6. Porcentaje de usuarios de internet

$33 \%$

$33 \%$

7. Suscripciones de banda ancha fija por cada 10 habitantes

8. Suscripciones de banda ancha móvil por cada 10 habitantes
5. Porcentaje de población que utiliza internet $33 \%$

6. Porcentaje de población con conexión $\quad 33 \%$ alámbrica

$33 \% \quad$ 7. Porcentaje de población con conexión $\quad 33 \%$ inalámbrica

\section{Factor: Capacidades para las TIC (20\% en el índice)}

9. Promedio de años de escolaridad

10. Tasa de matrícula de estudios de nivel secundaria

11. Tasa de matrícula de estudios de nivel terciario
$33 \%$

8. Porcentaje de población mayor a 18 años con estudios de primaria

$33 \%$ 9. Porcentaje de población mayor a 18 años con estudios secundarias (bachillerato)

$33 \%$ 10. Porcentaje de población mayor a 23 años $33 \%$ con estudios terciarios (nivel superior)

Fuente: Elaboración propia, 2020. 
A partir de la información de la ENDUTIH 2018 y siguiendo a Micheli y Valle (2018) se construyeron los 10 subfactores, utilizando la ecuación 1 para los subfactores de acceso y la ecuación 2 para los relacionados con uso y capacidades. En todos los casos, la elaboración de indicadores toma en cuenta el factor de expansión para cada variable.

$$
\begin{gathered}
\text { Subfactor }_{e}^{i}=\frac{\sum_{1}^{n} H_{n}^{i} * F E_{n}^{h} \mid i=1}{\sum_{1}^{n} H_{n} * F E_{n}^{h}} ; \forall H_{n} \in z \\
\text { Subfactor }_{e}^{i}=\frac{\sum_{1}^{n} P_{n}^{i} * F E_{n}^{p} \mid i=1}{\sum_{1}^{n} H_{n} * F E_{n}^{p}} ; \forall P_{n} \in z
\end{gathered}
$$

Donde:

Subfactor ${ }_{e}=$ subfactor de característica $i$ en la zona $z$.

$e=$ Variable dicotómica con 1 como respuesta afirmativa.

$H_{n}^{i}=$ Hogar de la muestra $n$ que presenta la característica $i$.

$F E_{n}^{h}=$ Factor de expansión correspondiente al hogar encuestado $n$.

$H_{n}=$ Hogar encuestado de la muestra $n$.

$P_{n}^{i}=$ Habitante de la muestra $n$ que presenta la característica $i$.

$F E_{n}^{p}=$ Factor de expansión correspondiente al habitante encuestado $n$.

$H_{n}=$ Habitante encuestado de la muestra $n$.

Con los valores obtenidos de los subfactores se calcularon los factores para cada estado $e$, usando la expresión 3. Cabe mencionar que se normalizaron los datos para garantizar que el conjunto de datos utilice la misma unidad de medida.

$$
\text { factor }_{e}^{i}=\sum_{1}^{i}\left(\text { Subfactor }_{e}^{i} * w_{i}\right)
$$

Donde:

factor $_{e}^{i}=$ factor del conjunto de características $i$ en el estado $e$. 
$i=$ Conjunto de características evaluadas: acceso, uso y capacidades.

$w_{i}=$ Peso de cada característica $i$ en factor ${ }^{i}$

A partir de los factores se obtuvo el IDT para el sector urbano y rural por entidad federativa de la siguiente forma:

$$
I D T=\sum_{1}^{i} \text { factor }_{e}^{i} * W_{i}
$$

Donde:

$W_{i}=$ Peso del factor $i$ en el IDT.

\section{5. Resultados.}

\subsection{Panorama de la población urbana y rural en México.}

Se realizaron cinco estimaciones de las tasas de crecimiento poblacional por ámbito y periodo intercensal y conteos. Con el propósito de mostrar la dinámica poblacional estatal y por ámbito rural y urbano con el sistema de consulta de series históricas de INEGI.

$$
r=\frac{\ln P t-\operatorname{Ln} P o}{t}
$$

Donde:

$R=$ Tasa de crecimiento de la población por ámbito

$P t=$ Población por ámbito del último censo

$P o=$ Población por ámbito del primer censo

$T=$ Tiempo transcurrido de último censo al primero

De acuerdo con las estimaciones, la tasa urbana nacional de mayor crecimiento poblacional fue durante el periodo 1980-2010 de alrededor de $2.78 \%$. Mientras que la de mayor crecimiento rural 
durante el periodo 2005-2010 con 1.6\%, no obstante, en todos los periodos siempre fue mayor la urbana. Para el periodo 2005-2010 los estados con mayor tasa de crecimiento urbana fueron Baja California Sur, Quintana Roo y Nayarit. En el ámbito rural Baja California, Ciudad de México (CdMX) y Morelos. La gráfica 1 proporciona un panorama de distribución estatal por ámbito para el año 2018 en virtud de que no hay diferencia significativa entre un año y otro se omitió el 2017. Las estimaciones son a partir de la tasa de crecimiento de población del periodo 2005-2010 y se interpola al año 2018, con la siguiente ecuación:

$$
P t=P o e^{r t}
$$

Se observa el peso significativo que la población urbana tiene en la mayoría de las entidades federativas. La participación porcentual indica que la CdMX, Nuevo León y Quintana Roo tienen al menos el $92 \%$ de la población estatal total en zonas urbanas. En tanto estados como Hidalgo, Chiapas y Oaxaca disponen de mayor población rural respecto del total, con un rango que va de $48 \%$ a $52 \%$.

Gráfica 1

Población por entidad según ámbito rural y urbano, 2018 (\%)

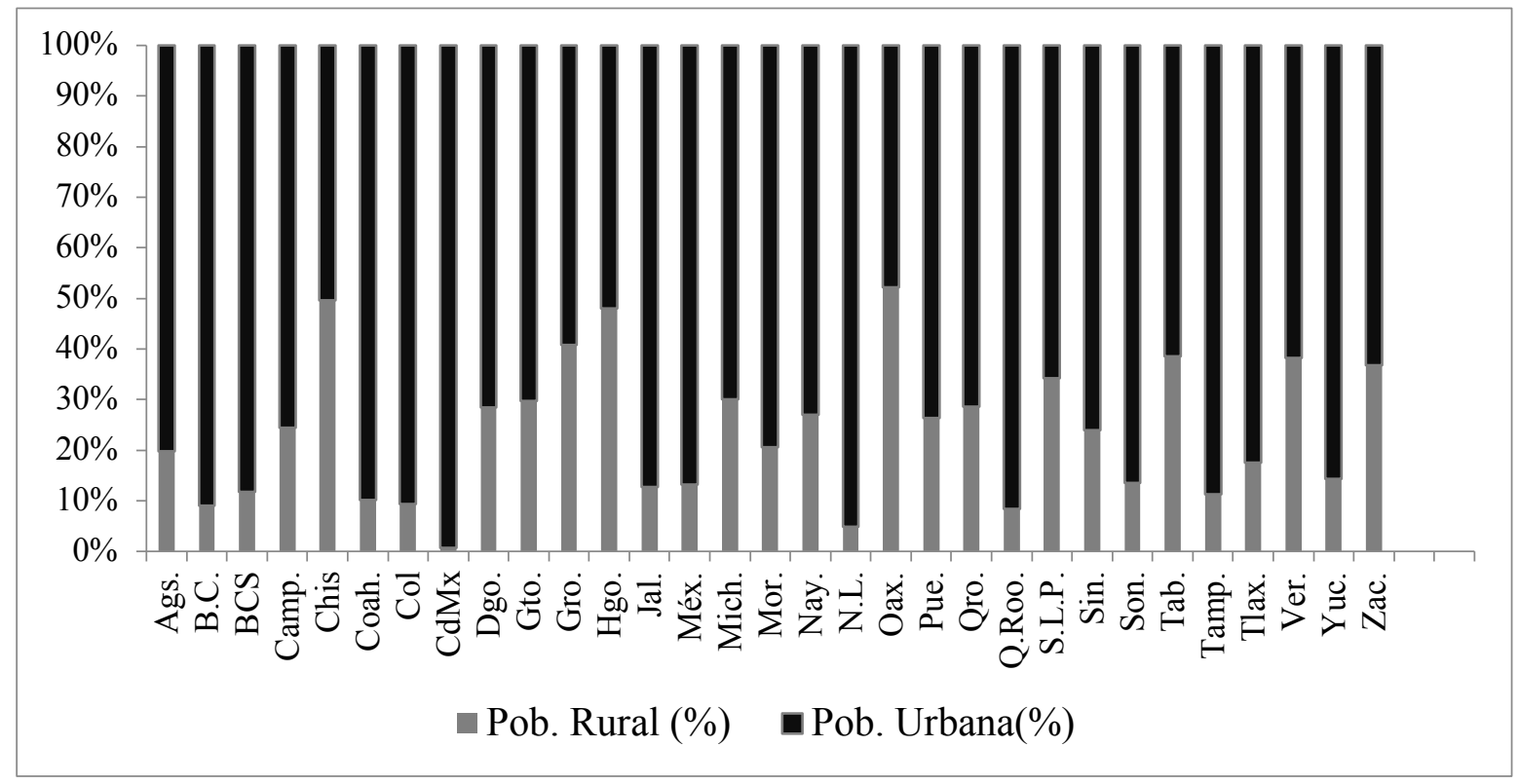

Fuente: Elaboración propia con serie histórica INEGI, 1990, 1995, 2005 y 2010. 


\subsection{Resultados del índice de desarrollo de las TIC a escala urbana y rural por estado.}

El índice permite comparar las zonas urbanas y rurales de las entidades del país para proponer una geografía de desarrollo de las TIC. Con respecto al sector urbano, existen estados como Guanajuato y Estado de México con potencial industrial y económico ubicados en la parte media baja del índice, lo contrario ocurre con Quintana Roo y Campeche del sureste que presentan valores superiores (ver gráfica 2). Sonora, CdMX y Baja California Sur encabezan los tres primeros lugares del índice de desarrollo de TIC en ambos años de la encuesta.

En el ámbito rural, la gráfica 3 muestra que los estados de Baja California, Sonora, Baja California Sur, Sonora y Morelos destacan con mayores puntajes en el índice. Sin embargo, Puebla y Coahuila con gran potencial económico se ubican en la parte baja de la medición, mientras entidades como Chiapas y Oaxaca se ubican por encima de los anteriores. Estos hallazgos reflejan que, en materia de desarrollo y apropiación de TIC, México no presenta la misma geografía que en los indicadores comúnmente utilizados y que dividen al país en una región norte moderna y desarrollada y el sur con un bajo nivel de desarrollo. El estado de Tlaxcala es el que se encuentra más rezagado en cuanto al acceso y uso de las tecnologías digitales.

Gráfica 2

Valor del IDT urbano por entidad federativa, 2017 y 2018

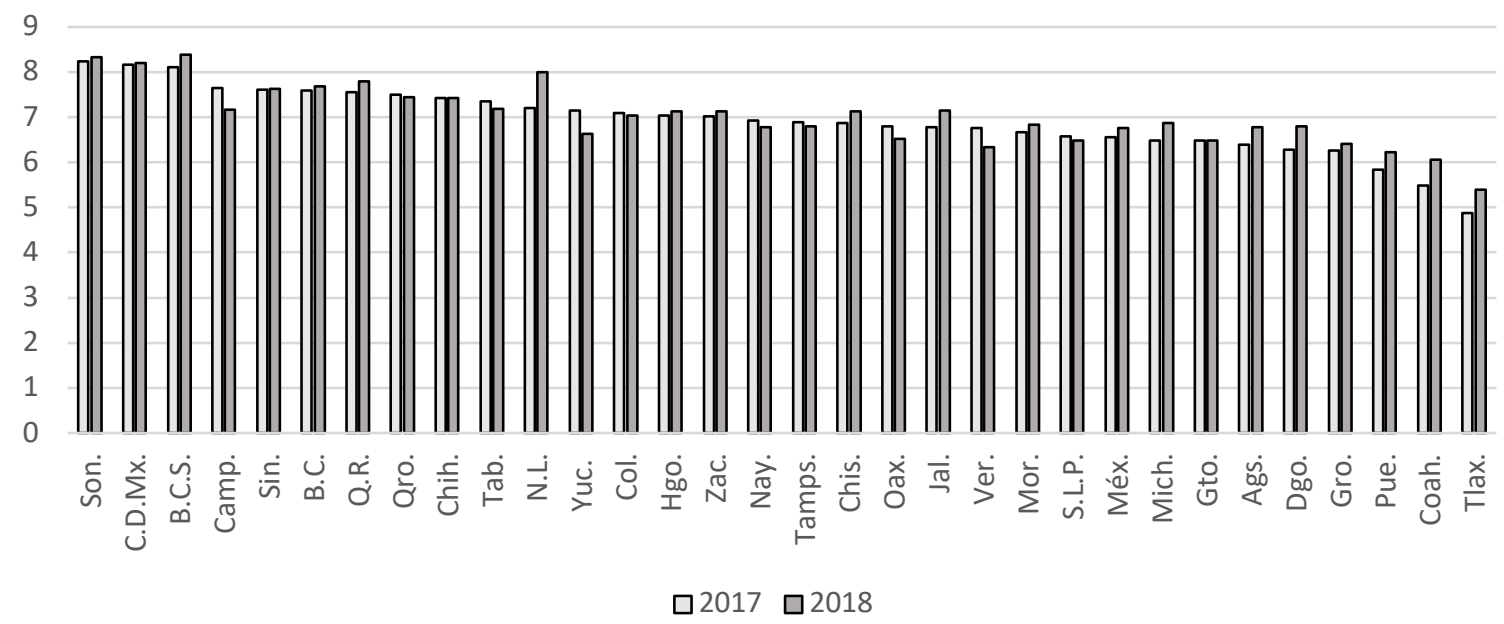

Fuente: Elaboración propia con datos de la ENDUTIH, 2018. 
Gráfica 3

Valor del IDT urbano por entidad federativa, 2017 y 2018

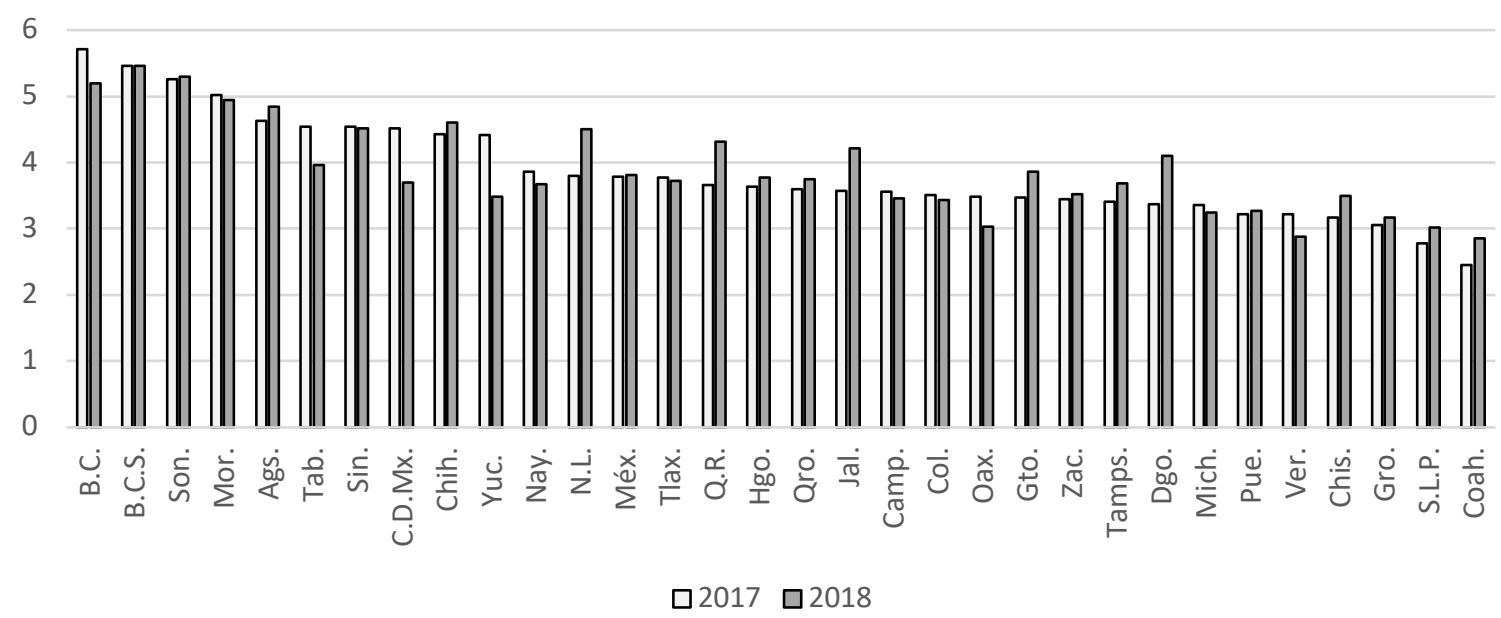

Fuente: Elaboración propia con datos de la ENDUTIH, 2018.

En la gráfica 4 se visualizan los puntajes de cada uno de los componentes del índice por entidad y ámbito según año. Para las zonas urbanas, los estados con mayor infraestructura de TIC o conectividad son los que contienen las principales ciudades del país como CdMX, Nuevo León, Baja California y Jalisco. En tanto que los usuarios urbanos de TIC se ubican en mayor medida en Sonora y Quintana Roo. En capacidades, relacionada con la educación de la población, los valores más altos los presenta Ciudad de México.

Con relación al sector rural, en la gráfica 4 se observa que los estados que presentan mayor conectividad fueron Baja California, Sonora y Morelos. En el uso, se reflejan diferencias significativas entre ambos años de la encuesta, sin embargo, destacan Baja California y Sonora y, por el contrario, los últimos lugares lo ocupan Coahuila y San Luis Potosí. Finalmente, en cuanto a capacidades sobresale Sinaloa. 


\section{Valor del IDT por entidad federativa, según ámbito y componente del índice}

(Acceso, Uso y Capacidades)

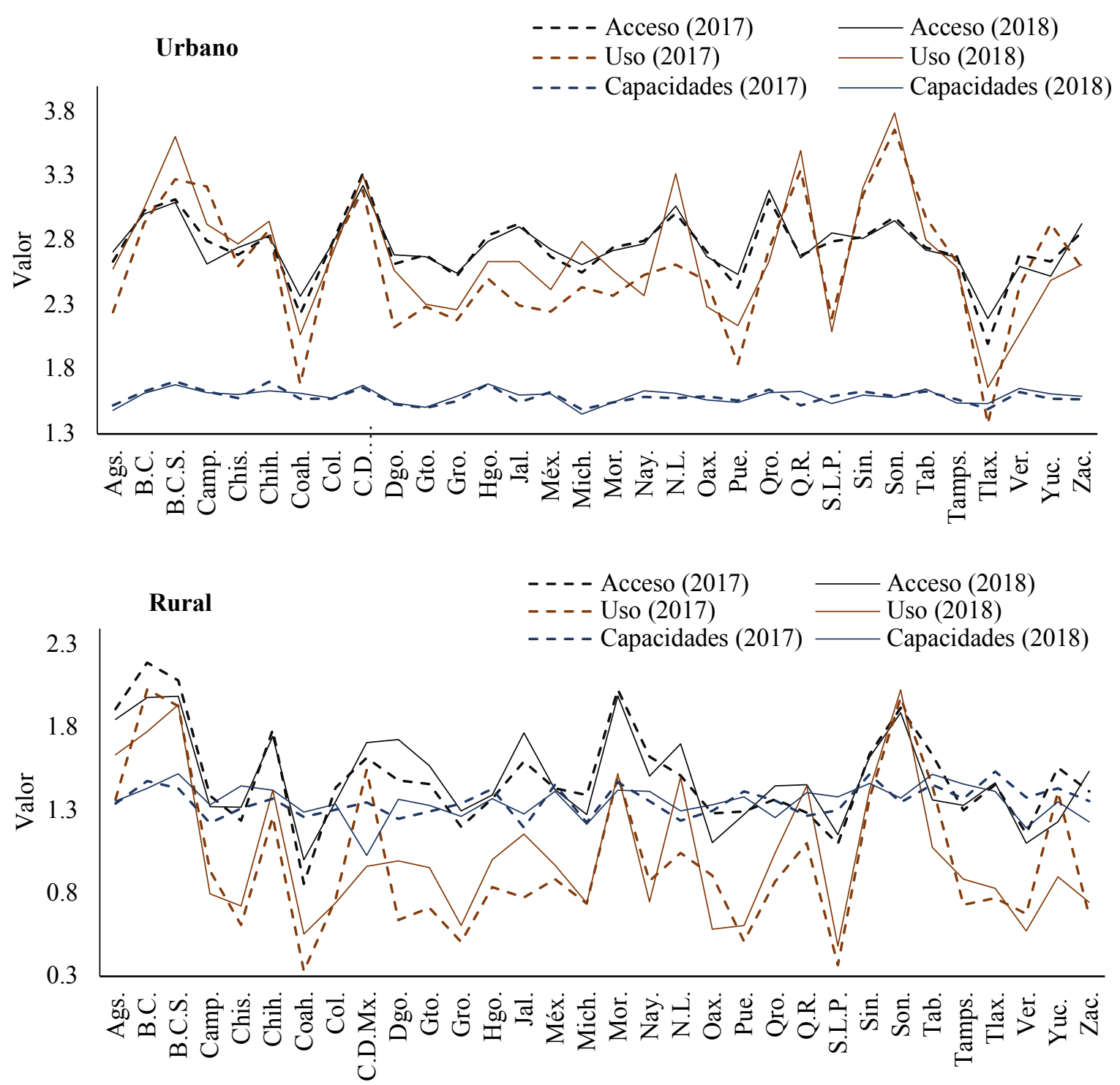

Fuente: Elaboración propia con datos de la ENDUTIH, 2017 y 2018.

El índice como vector de la apropiación social de tecnologías digitales muestra la tendencia general de la terciarización de la economía mexicana. Los resultados de la regionalización digital permiten comprender la geografía económica mexicana a la luz de la reestructuración productiva en la que se incorpora los servicios. Las capacidades digitales deben compararse con el aporte del sector terciario al perfil productivo. Para analizar esta relación, se estiman regresiones por separado entre el índice de desarrollo de las TIC y la estructura laboral (en sector secundario y terciario) sólo para el 
ámbito urbano, debido a la no disponibilidad de información sobre población ocupada en el sector rural.

El objetivo de la regresión es validar la consistencia del IDT urbano con la estructura laboral de la Encuesta Nacional de Ocupación y Empleo (ENOE). Cabe mencionar que se toma como variable proxy el porcentaje de población ocupada en el sector secundario y terciario (INEGI, 2017 y 2018b). Como se observa en la gráfica 5, el nivel de apropiación de las TIC no guarda relación con el sector secundario, pero sí con el terciario.

\section{Gráfica 5}

\section{IDT urbano según entidad federativa y porcentaje de personal} ocupado en el sector secundario

2017 y 2018
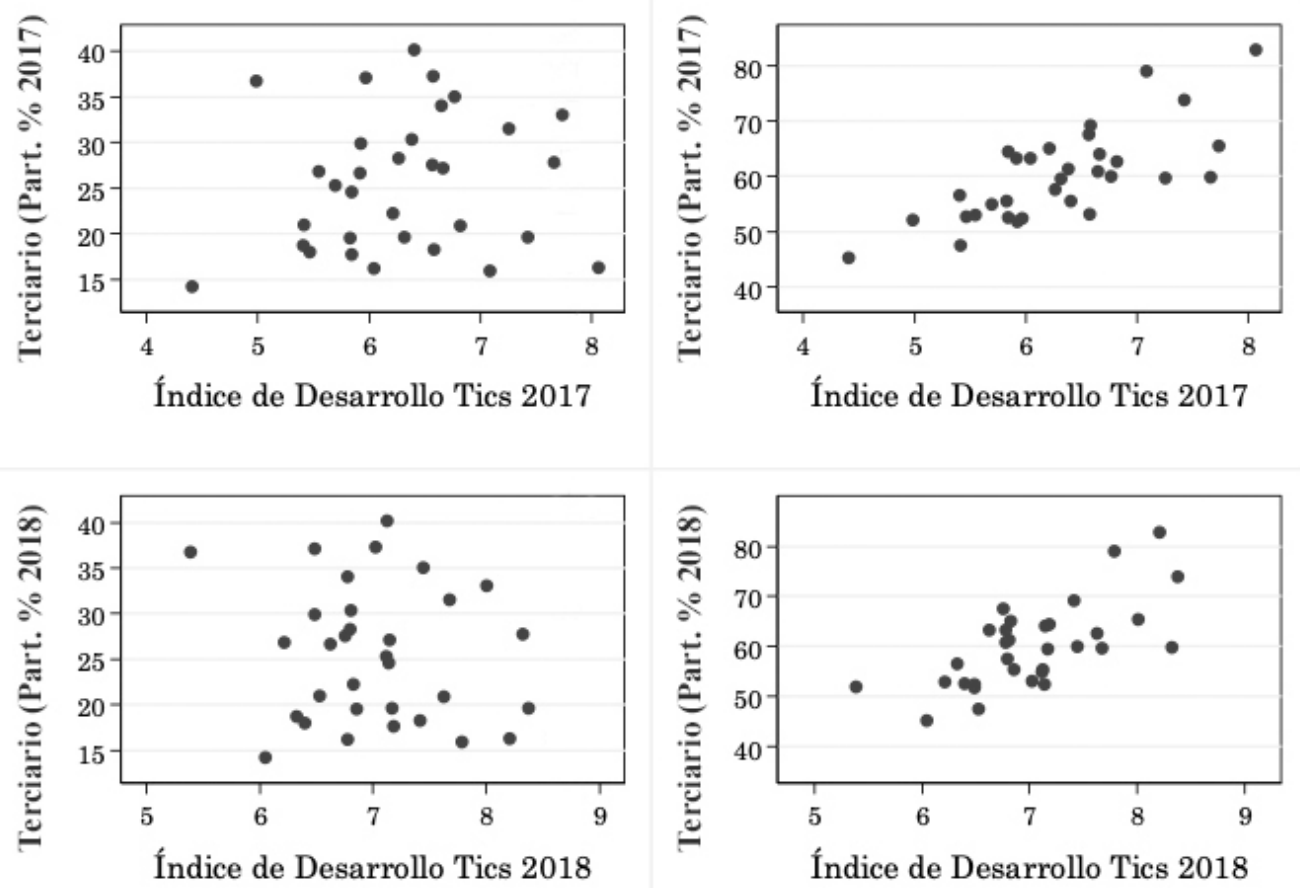

Fuente: Elaboración propia con datos de la ENDUTIH y ENOE, 2017 y 2018.

Los resultados del índice de desarrollo de TIC a escala urbana no se relacionan con el porcentaje de población ocupada en el sector secundario, pero sí con el porcentaje de población ocupada en el sector comercio y servicios. Con base en los resultados de la estimación de la regresión 
lineal entre las variables, considerando el ámbito urbano de los 32 estados del país, se confirma que el índice de desarrollo de TIC es estadísticamente representativo para explicar el porcentaje de población ocupada en el sector terciario, según el resultado de la prueba $\mathrm{t}(<0.05)$, así como un $\mathrm{R}$ cuadrada por encima del 50\% (ver cuadro 7). De igual forma se realiza el ejercicio respecto al porcentaje de personal ocupado en el sector secundario, sin embargo, los resultados no son estadísticamente significativos.

\section{Cuadro 4}

\section{Estimación lineal de la relación del porcentaje de población ocupada en sector terciario e Índice de Desarrollo de TIC}

\begin{tabular}{|c|c|c|c|c|}
\hline & 2017 & & 2018 & \\
\hline Variables & $\begin{array}{l}\text { Coeficiente (error } \\
\text { estándar) }\end{array}$ & $P>|t|$ & $\begin{array}{l}\text { Coeficiente } \\
\text { (error estándar) }\end{array}$ & $\mathrm{P}>|\mathrm{t}|$ \\
\hline Índice de desarrollo de TIC & $\begin{array}{l}0.0777124 \\
(0.0127453)\end{array}$ & $0^{* * *}$ & $\begin{array}{l}0.0851815 \\
(0.0168042)\end{array}$ & $0 * * *$ \\
\hline Constante & $\begin{array}{c}0.1094435 \\
(0.0810466)\end{array}$ & 0.19 & $\begin{array}{l}0.0008081 \\
(.1186733)\end{array}$ & 0.99 \\
\hline
\end{tabular}

***Significativo al $1 \%$.

Fuente: Elaboración propia 2020.

\section{6. Conclusiones.}

La brecha digital es una desigualdad adicional a las condiciones económicas y sociales. Las estadísticas internacionales ubican al país en los últimos lugares en el contexto latinoamericano, pues no ha logrado asociar su dimensión económica con la apropiación de las TIC por parte de los habitantes. Tanto el IDT urbano como el IDT rural reflejan que el país es muy heterogéneo, entidades que son relevantes debido a su crecimiento económico tienen una debilidad en materia de la sociedad de la información. De igual forma, los estados con mayor población rural a nivel estatal disponen de índices IDT bajos, mientras estados con mayor población urbana a nivel estatal disponen de índices IDT altos. Por otro lado, la evaluación de la consistencia del índice de TIC urbano con la estructura laboral local permitió identificar la asociación entre el sector terciario y la brecha digital, la cual es multidimensional y multifactorial. Asimismo, la ubicación del país en el contexto internacional de la sociedad de la información requiere centrar la atención en el contexto local, en el que se desarrollen las potencialidades de los espacios, para inscribirlos en la sociedad de la información y el 
conocimiento. La limitante del presente trabajo es la no disponibilidad de información sobre población rural ocupada para establecer la relación entre el Índice de desarrollo de TIC rural y las personas empleadas en el sector secundario y terciario. 


\section{Referencias bibliográficas y documentales}

Aker, J. C., Ghosh, I., y Burrell, J. (2016). "The promise (and pitfalls) of ICT for agriculture initiatives." Agricultural Economics,núm. 47(S1), pp. 35-48. https://doi.org/10.1111/agec.12301

Ayala, E., Chapa, J., García, L., y Hibert, A. (2018). "Welfare effects of the telecommunication reform in Mexico." Telecommunications Policy, vol. 42, núm. 1, pp. 24-36. https://doi.org/10.1016/j.telpol.2017.07.013

Blank, G., y Groselj, D. (2014). "Dimensions of Internet use: amount, variety, and types." Information, Communication \& Society, vol. 17, núm. 4, pp. 417-435. https://doi.org/10.1080/1369118X.2014.889189

De la Selva, A. R. A. (2015)." Los nuevos rostros de la desigualdad en el siglo XXI: la brecha digital." Revista Mexicana de Ciencias Políticas y Sociales, vol. 60, núm. 223, pp. 265-285. https://doi.org/10.1016/S0185-1918(15)72138-0

Dewan, S., y Riggins, F. J. (2005). "The digital divide: Current and future research directions." Journal of the Association for Information Systems, vol. 6, núm. 12, pp. 298-337.

Diario Oficial de la Federación 13 de Junio. (2013). "Reforma del artículo 6o constitucional." Disponible en http://www.diputados.gob.mx/LeyesBiblio/ref/dof/CPEUM_ref_208_11jun13.pdf

Felizzola, M. 2010. "Tecnologías de información y comunicación para el desarrollo rural en Colombia." Economía Gestión y Desarrollo, núm. 10, pp. 97-124. Recuperado de https://revistas.javerianacali.edu.co/index.php/economia/article/view/387

Gobierno de la República (2013). "Estrategia Digital Nacional”. Disponible en http://cdn.mexicodigital.gob.mx/EstrategiaDigital.pdf

Goncalves, G., Oliveira, T., y Cruz-Jesús, F. (2018). "Understanding individual-level digital divide: Evidence of an African country." Computers in Human Behavior, núm. 87, pp. 276-291. https://doi.org/10.1016/j.chb.2018.05.039

Gunkel, D. J. (2003). "Second thoughts: toward a critique of the digital divide." New Media \& Society, vol. 5, núm. 4, pp. 499-522. https://doi.org/10.1177/146144480354003

Gwaka, L. T., May, J., y Tucker, W. (2018). "Towards low cost community networks in rural communities: The impact of context using the case study of Beitbridge, Zimbabwe." The Electronic Journal of Information Systems in Developing Countries, vol. 84, núm. 3, pp. 1-11. https://doi.org/10.1002/isd2.12029

Helsper, E. J. (2010). “Gendered internet use across generations and life stages.” Communication Research, vol. 37, núm. 3, pp. 352-374. https://doi.org/10.1177/0093650209356439

Hodge, H., Carson, D., Carson, D., Newman, L., y Garrett, J. (2017). "Using Internet technologies in rural communities to access services: The views of older people and service providers." Journal of Rural Studies, núm. 54, pp. 469-478. https://doi.org/10.1016/j.jrurstud.2016.06.016

INEGI (2017). "Encuesta Nacional sobre Disponibilidad y Uso de Tecnologías de la Información y Comunicación en los Hogares 2017." Disponible en www.inegi.org.mx

INEGI (2018a). "Encuesta Nacional sobre Disponibilidad y Uso de Tecnologías de la Información y Comunicación en los Hogares 2018." Disponible en www.inegi.org.mx

INEGI (2018b). "Encuesta Nacional sobre Ocupación y Empleo 2018.” Disponible en www.inegi.org.mx

INEGI (2020). "Consulta Interactiva de Conteos y Censos de Población Serie Histórica." Disponible en https://www.inegi.org.mx/programas/ccpv/cpvsh/

ITU (2018). "Measuring the information society report 2018." Disponible en https://www.itu.int/en/ITUD/Statistics/Documents/publications/misr2018/MISR-2018-Vol-1-E.pdf

Malecki, E. J. (2003). "Digital development in rural areas: potentials and pitfalls." Journal of Rural Studies, vol. 19, núm. 2, pp. 201-214. https://doi.org/10.1016/S0743-0167(02)00068-2 
Mariscal, J. (2005). “Digital divide in a developing country.” Telecommunications Policy, vol. 29, núm. 5-6, pp. 409-428. https://doi.org/10.1016/j.telpol.2005.03.004

Márquez, A. M., Acevedo, M. A., y Castro, L. D. (2016)."Brecha digital y desigualdad social en México." Economía Coyuntural, Revista de Temas de Perspectivas y Coyuntura, vol.1, núm. 2, pp. 89-136.

Martínez-Domínguez, M. (2018). “Acceso y uso de tecnologías de la información y comunicación en México: factores determinantes." PAAKAT: Revista de Tecnología y Sociedad, vol. 8, núm.14. http://dx.doi.org/10.32870/Pk.a8n14.316

Micheli, J. y Valle (2018). "La brecha digital y la importancia de las tecnologías de la información y la comunicación en las economías regionales de México." Realidad, datos y espacio Revista Internacional de Estadística y Geografía, vol. 9, núm. 2, pp. 38-53. Recuperado de http://internet.contenidos.inegi.org.mx/contenidos/sitios/rdebeta/rde_26/RDE25_art04.pdf

Mossberger, K., Tolbert, C. J., y Stansbury, M. (2003). Virtual inequality: Beyond the digital divide. Georgetown University Press.

Nishijima, M., Ivanauskas, T. M., y Sarti, F. M. (2017). "Evolution and determinants of digital divide in Brazil (2005-2013).” Telecommunications Policy, vol. 41, núm. 1, pp. 12-24. https://doi.org/10.1016/j.telpol.2016.10.004

OCDE (2001). "Understanding the digital divide." Disponible en www.oecd.org/sti/1888451.pdf

OCDE (2018). "Bridging the rural digital divide." Disponible en https://www.oecdilibrary.org/docserver/852bd3b9en.pdf?expires $=1567027027 \&$ id $=$ id\&accname $=$ guest\&checksum=B511A837C34B1F2DAF5FE9B6 3E7AE4D2

Ovando, C., y Olivera, E. (2018). "Was household internet adoption driven by the reform? Evaluation of the 2013 telecommunication reform in Mexico." Telecommunications Policy, vol. 42, núm. 9, pp. 700-714. https://doi.org/10.1016/j.telpol.2018.03.005

Palvia, P., Baqir, N., y Nemati, H. (2018). "ICT for socio-economic development: A citizens' perspective." Information \& Management, vol. 55, núm. 2, pp. 160-176. https://doi.org/10.1016/j.im.2017.05.003

Pressat R. (1983). Los métodos en Demografía. Oikus-Tau.

Szeles, M. R. (2018). "New insights from a multilevel approach to the regional digital divide in the European Union." Telecommunications Policy, vol. 42. núm. 6, pp. 452-463. https://doi.org/10.1016/j.telpol.2018.03.007

Ruiz, W. (2015). "Desigualdades entre entidades en materia de tecnologías de información y comunicación en México." Realidad, datos y espacio Revista Internacional de Estadística y Geografia, vol. 6, núm. 1, pp. 36-49. Recuperado de https://www.inegi.org. mx/rde/2015/01/07/desigualdades-entre-entidadesen-materia-de-tecnologias-de-informacion-y-comunicacion-en-mexico/

Salemink, K., Strijker, D., y Bosworth, G. (2017). "Rural development in the digital age: A systematic literature review on unequal ICT availability, adoption, and use in rural areas." Journal of Rural Studies, núm. 54, pp. 360-371. https://doi.org/10.1016/j.jrurstud.2015.09.001

Scheerder, A., Van Deursen, A., y Van Dijk, J. (2017). "Determinants of Internet skills, uses and outcomes. A systematic review of the second-and third-level digital divide." Telematics and Informatics, vol. 34, núm. 8, pp. 1607-1624. https://doi.org/10.1016/j.tele.2017.07.007

Selwyn, N. (2004). "Reconsidering political and popular understandings of the digital divide." New Media \& Society, vol. 6, núm. 3, pp. 341-362. https://doi.org/10.1177/1461444804042519

Tirado-Morueta, R., Mendoza-Zambrano, D. M., Aguaded-Gómez, J. I., y Marín-Gutiérrez, I. (2017). "Empirical study of a sequence of access to Internet use in Ecuador." Telematics and Informatics, vol. 34, núm. 4, pp. 171-183. https://doi.org/10.1016/j.tele.2016.12.012

Torero, M. y Von Braun, J. 2006. "Impacts of ICT on low-income rural households", en Maximo Torero y Joachim Von Braun (eds.), Information and communications technologies for development and poverty reduction, International Food Policy Research Institute (IFPRI), Washington, D. C. (pp. 234-311). 
Toudert, D. (2013). "La brecha digital en los contextos de marginación socioterritorial en localidades mexicanas: exploración y discusión."Comunicación y Sociedad, núm. 19, pp. 153-180.

Townsend, L., Sathiaseelan, A., Fairhurst, G., y Wallace, C. (2013). "Enhanced broadband access as a solution to the social and economic problems of the rural digital divide." Local Economy, vol. 28, núm. 6, pp. 580-595. https://doi.org/10.1177/0269094213496974

Van Deursen, A. J., y Helsper, E. J. (2015). "The third-level digital divide: Who benefits most from being online?." Communication and Information Technologies Annual (pp. 29-52). Emerald Group Publishing Limited. https://doi.org/10.1108/S2050-206020150000010002

Van Deursen, A. J., Helsper, E., Eynon, R., y Van Dijk, J. A. (2017). "The compoundness and sequentiality of digital inequality.” International Journal of Communication,núm. 11, pp. 452-473. Recuperado de http://eprints.1se.ac.uk/68921/

Van Deursen, A. J., y Van Dijk, J. A. (2014). "The digital divide shifts to differences in usage.” New Media \& Society, vol. 16. núm. 3, pp. 507-526. https://doi.org/10.1177/1461444813487959

Van Dijk, J. A. (2006). "Digital divide research, achievements and shortcomings."Poetics, vol. 34, núm. 4-5, pp. 221-235. https://doi.org/10.1016/j.poetic.2006.05.004

Van Dijk, J., y Hacker, K. (2003). "The digital divide as a complex and dynamic phenomenon." The Information Society, vol.19, núm. 4, pp. 315-326. https://doi.org/10.1080/01972240309487

Whitacre, B. E., y Mills, B. F. (2007). "Infrastructure and the rural-urban divide in high-speed residential Internet access." International Regional Science Review, vol. 30, núm. 3, pp. 249-273. https://doi.org/10.1177/0160017607301606 


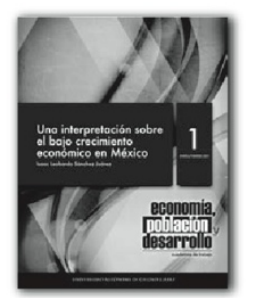

Economia, población y desarrollo.

Cuadernos de trabajo №1
Enero-Febrero 2011

Una interpretación sobre el bajo
crecimiento económico en México

crecimiento económico en México
Isaac Leobardo Sánchez Juárez
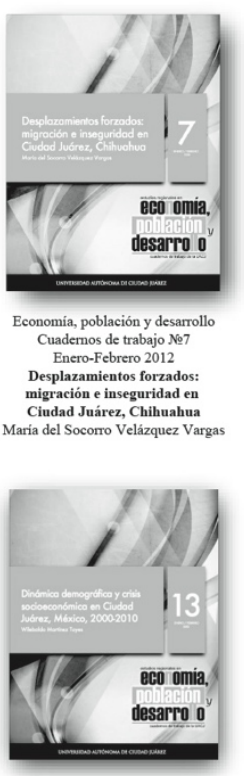

Economia, población y desarrollo Cuarednos de trabajo oㅡ
Enero - Febrero 2013

Dinámica demográfica y crisis
socieconómica en Ciudad Juárez,

México, 2000-2010
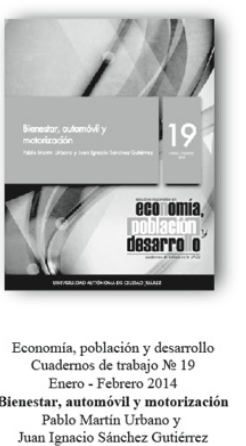

Pablo Martin Urbano y
Juan Ignacio Sánchez Gutiémez

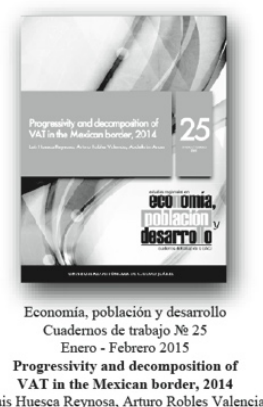

Abdelkim Araa
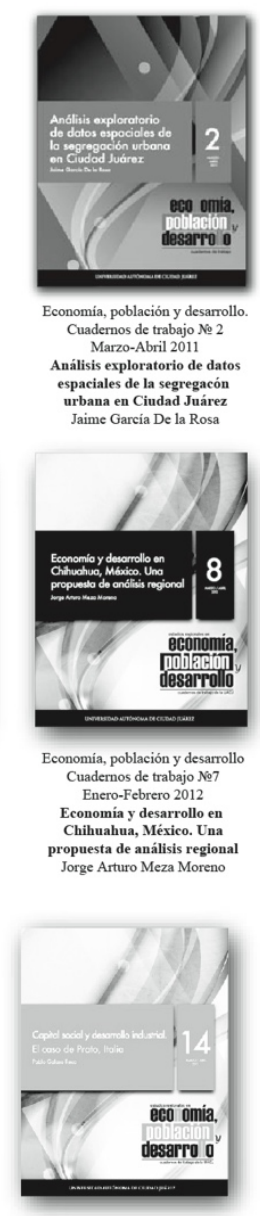

Economia, población y desarrollo

Cuadernos de trabajo №
Marzo - Abril 2013

Capital social y desarrollo
dustrial. El caso de Prato, Ital Pablo Galaso Reca
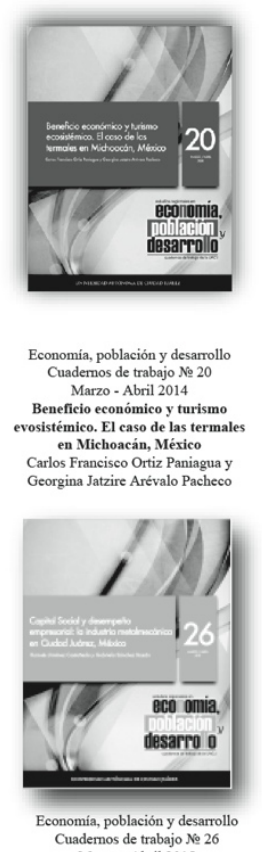

Cuadernos de trabajo № 26 Marzo - Abril 2015
Capital Social y desempeño empresa la industria metalmecánica en
Ciudad Juárez, México Ramsés Jiménez Castaneda y
Gabriela Sáncez Bazán

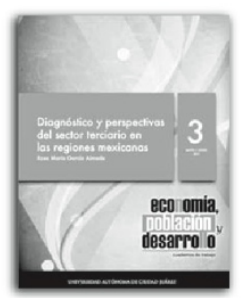

Economia, población y desarrollo. Cuadernos de trabajo № 3
Mayo-Junio 2011 Diagnóstico y perspectivas del sector terciario en las
regiones mexicanas

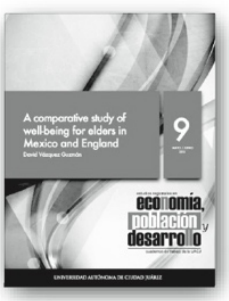

Economia, población y desarrollo Cuadernos de trabajo № 9
Mayo - Junio 2012 A comparative study of well-being for elders is
Mexico and England David Vázquez Guzmár
and

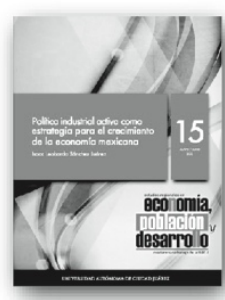

Economia, población y desarrollo Cuademos de trabajo $\mathrm{NO}^{\circ}$
Mayo - Junio 2013 Politica industrial activa como estrategia para el crecimiento Isaac Leobardo Sánchez Juárez

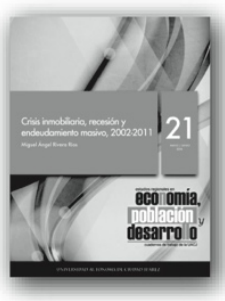

Economia, población y desarrollo

Cuadernos de trabajo
Mayo - Junio 2014

Crisis inmobiliaria, recesión y
endeudamiento masivo, 2002 -2011

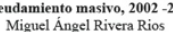

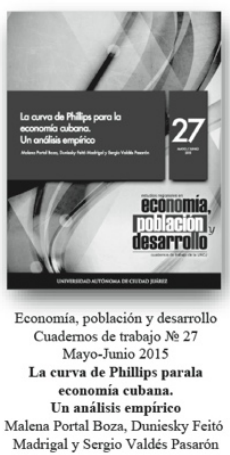

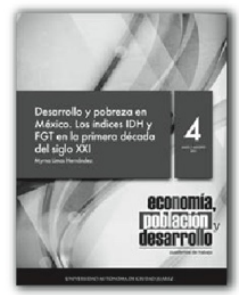

Economiá, población y desarrollo. julio-Agosto 2011

Dasarrollo y pobreza en México. Los indices IDH y FGT en la Myrna Limas Hernández

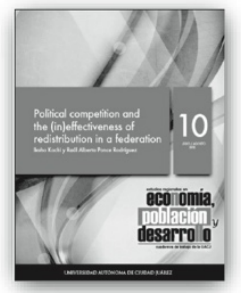

Economiá, poblacín y decarrollto Cuademos de trabajo № 10
Julio - Agosto 2012 Political competition and the (ii)effectiveness of redistribution in a federation
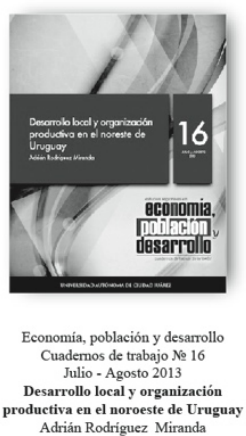
Cuadernos de trabajo № 17
Septiembre - Octubre 2013 erabilidad social y vivi en Sonora, Mexico
Jesús Enriquez Acosta

Cuadernos de trabajo № 11 Septiembre - Octubre 2012
Análisis y determinantes de la productividad legislativa Bárbara Briones Martinez

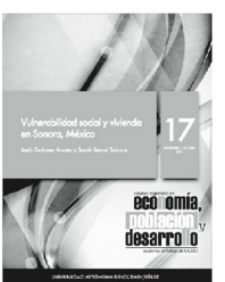

uctiva en el noroeste de Urug Sarah Bernal Salazar
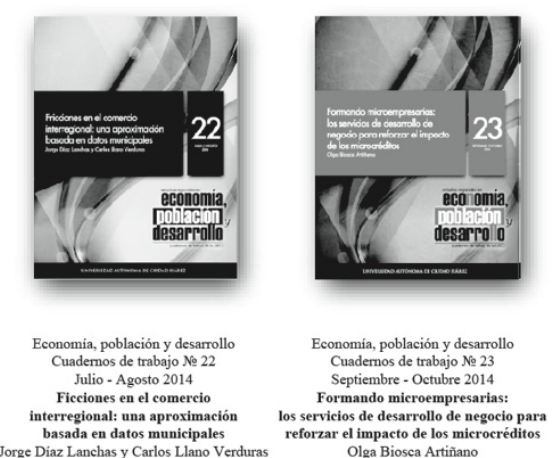
te los microcrédito
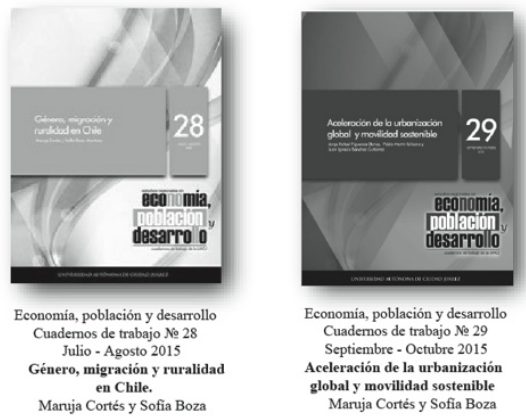

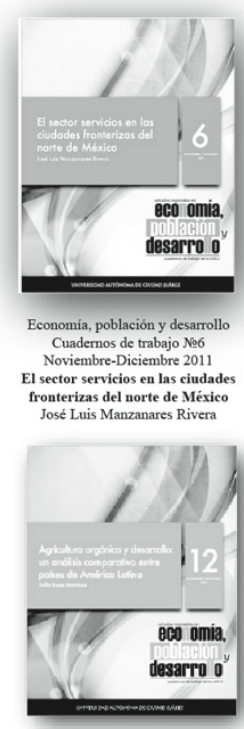

Economia, población y desarrollo Cuademos de trabajo № 12
Noviembre - Diciembre 2012 griculitura organica y desarrolle: Sofia Boza Martinez

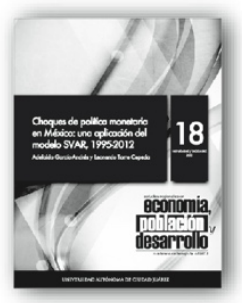
Noviembre - Diciembre 2013 Choques de politica monetaria
en México: una aplicación del do Garcia-Andés y L

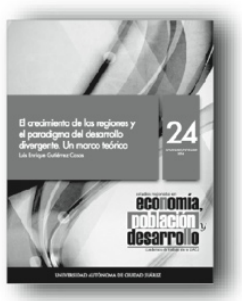

Economia, población y desarrollo Cuadernos de trabajo № 24 El crecimiento de las regiones $y$ el paradigma del desarrollo Luis Enrique Gutiérez Casas

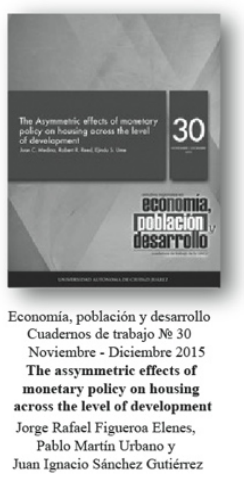



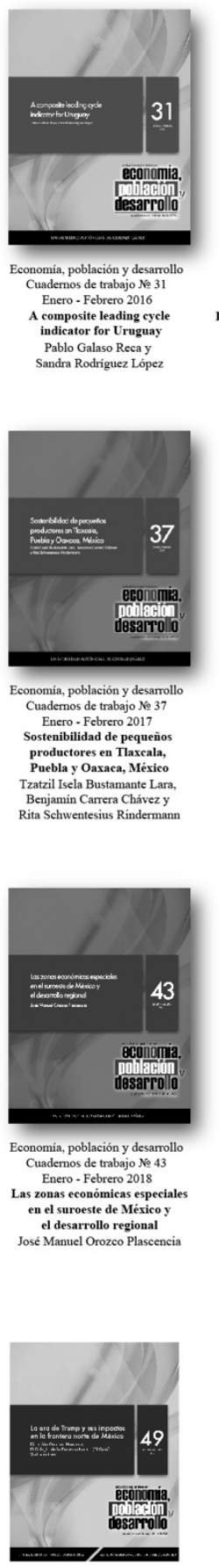

Economia, población y decararollo
Cuademos de trabajo Ne 49 Enero- Febrero 2019

La era de Trump y sus impactos
en la frontera norte de México

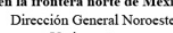
Varios autors
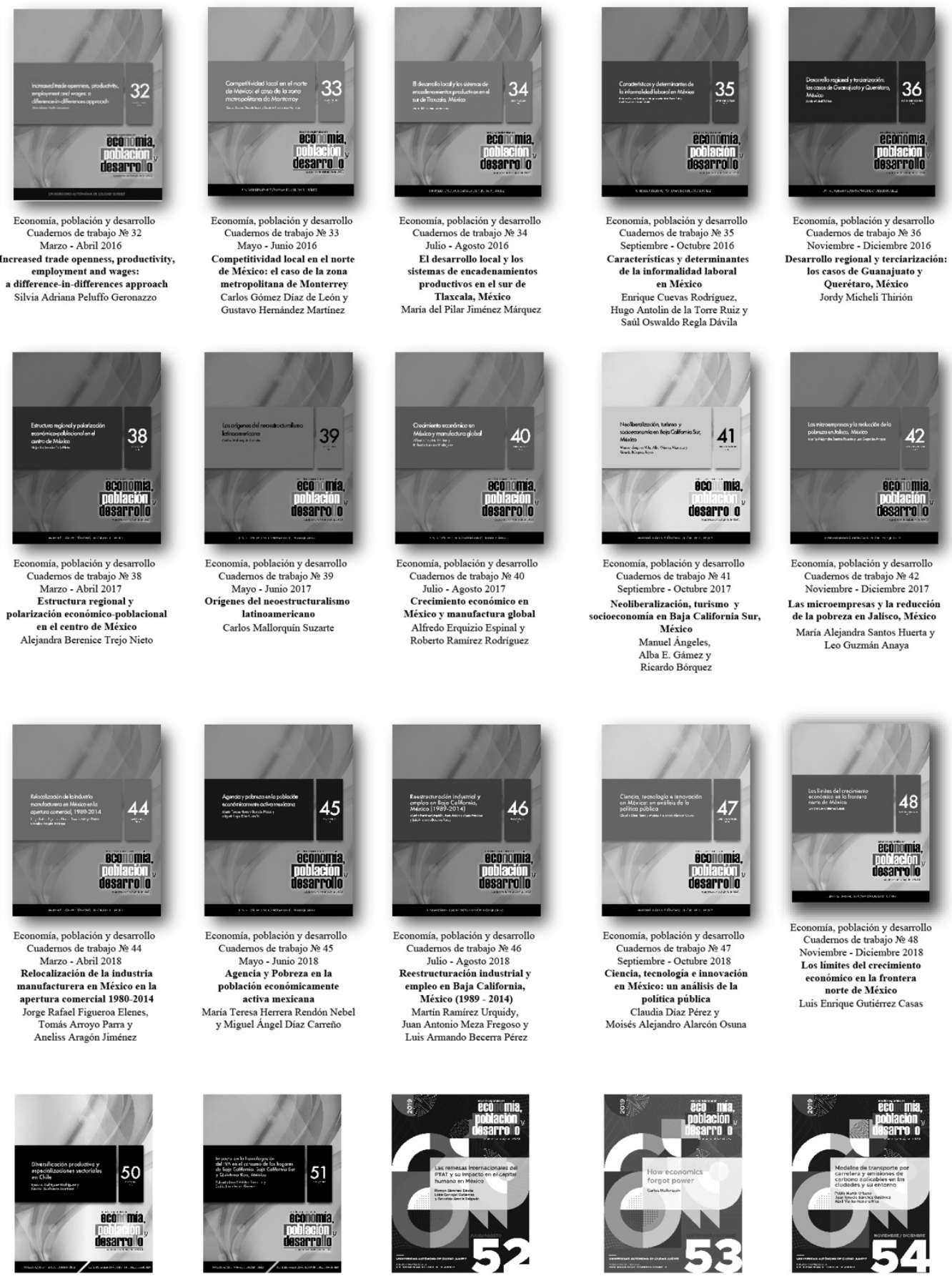

Economia, población y desaraollo
Cuademos de trabajo No 50 Marzo-Abril 2019 Diversificación productiva
especializaciones sectoriales en Chile Ignacio Rodriguez Rodriguez
Pallina Sanhlueza Martinez

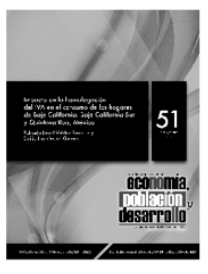

Economia, población y desarrollo
Cuadernos de trabajo No 51 Mayo-Junio 2019 Impacto de la homologación del IVA
en el consumo de los hogagres
Baja Califioria, Baja Califireria Sur $y$ Quintana Roo, México Rolando Irrael Valdez Ramirezy
Emilio Hemandez Gómez
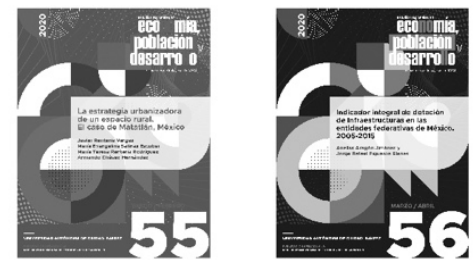

Economia, población y desarmollo
Cundenos ate Cuademos de trabajo No 93
Enero - Febrero 2020 Enero - Febrero 2020
La estrategia urbanizadora
de un expacio rural. de un espacio rural
El casso de Matatalin, Mexico. Javier Renteria Vargas, Maria Evangeclina Salinas Escobar
Maria Teress Renteria Rodriguez Armando Chávez Hemández
Economia, población n decsarollo
Cundemes dict trabajai Cuademos de trabajo
Marzo-Abril 2020

Indicaador integral de dotación de
infraestructuras en las entidade fraestructuras en las entidade
federativas de Mexico,

Ancliss Aragón Jiménez y
Jorge Rafacl Figueroa Elenes

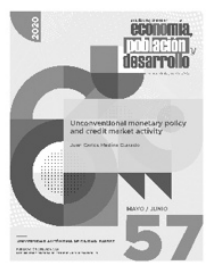

Economia, población y desarrollo Mayo - Junio 2020

Unconventional monetary policy
and creditmarket activity Juan Carlos Medina Guirado

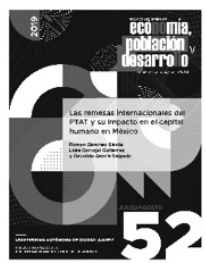

Economia, población y desarrollo
Cuademos de trabajo No 52 Julio - Agosto 201

Las remesas internacionales
del PTAT y su impacto en

Román Śánchez Dávila
Lidia Carvajal Gutiérez y
Oswaldo Garcia Sal gado

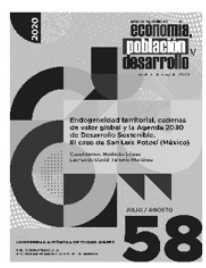

Economiá, población y desarrollo Julio - Agosto 202 Endogeneidad territorial,
adenas de valor global $y$ cadenas de valor global y la
Agenda 2030 de Desarrollo Sostenib El caso de San Luis Potosí (México) Cuauhtémoc Modesto López y
Leonardo David Tenorio Martinez

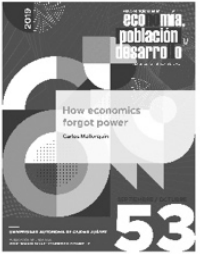

Economia, población y desarrollo Cuademos de trabajo № 53 How economics forgot power
Carlos Mallorquin

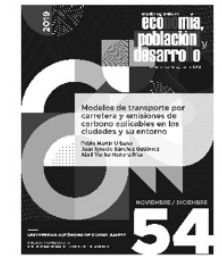

Economia, población y desarrollo
Cuadernos de trabajo № 54 Modelos de transporte por carreter Modelos de transporte por carretera
emisiones de carbono aplicables en las ciudades $y$ su entorn Juan Ignacio Sánchez Gutiérrez y Abril Yuriko Herrera Rios

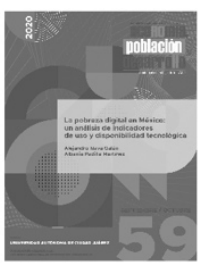

Economia, población y desarrollo Septiembre - Octubre 2020 La pobreza digital en México: análisis de indicadores de uso
disponibilidad tecnologica Alejandro Nava Galán y 


\section{Para el documento general:}

Tipo de letra: Times New Roman.

Tamaño: 11 puntos.

Interlineado: 1.5 espacios.

Títulos y subtítulos:

El texto principal en 11 puntos. Títulos 12 puntos (en resaltado). Subtítulos 11 puntos. Cada título y subtítulo deberá numerarse bajo el siguiente orden: 1, 1.1, 2, 2.1, 2.2...

La extensión máxima de los cuadernos de trabajo será de 40 cuartillas.

La primera vez que se emplee una sigla en el texto se especificará primero su equivalencia completa y después la sigla.

\section{Hoja de presentación:}

Título:

14 puntos, centrado, resaltado.

Nombre de autor(es):

12 puntos

Resumen y abstract:

Debe incluir resumen en español y abstract (diez puntos), no mayor a 250 palabras

Palabras clave:

Incluir entre tres y cinco palabras clave, en español e inglés

Referencia del autor o autores:

Institución de adscripción, grado académico y líneas-grupos de investigación que desarrolla y a los que pertenece.

\section{Sistema de referencia de citas:}

Harvard-APA

Las citas bibliográficas en el texto deberán incluir entre paréntesis sólo el apellido del autor, la fecha de publicación y el número de página; por ejemplo: (Quilodrán, 2001: 33).

\section{Notación en sección de bibliografía y fuentes de información:}

Se deberá incluir al final del texto. Toda referencia deberá estar mencionada en el texto o notas de pie de página.

Cada referencia iniciará con el primer apellido o los apellidos, luego el nombre del autor, y después, entre paréntesis, el año de publicación seguido de un punto. Ejemplos:

Se deberá incluir al final del texto. Toda referencia deberá estar mencionada en el texto o notas de pie de página.

Cada referencia iniciará con el primer apellido o los apellidos, luego el nombre del autor, y después, entre paréntesis, el año de publicación seguido de un punto. Ejemplos: 
Artículo:

Ros, Jaime (2008). "La desaceleración del crecimiento económico en México desde 1982", en Trimestre Económico, vol. 75, núm. 299, pp. 537-560.

Libro:

Villarreal, René (2005). Industrialización, competitividad y desequilibrio externo en México.

Un enfoque macroindustrial y financiero (1929-2010), México, Fondo de Cultura Económica.

Capítulo de libro:

Castillo, Manuel Ángel (2003). "La política de inmigración en México: un breve recuento", en Manuel Ángel Castillo, Alfredo Lattes y Jorge Santibáñez (coords.), Migración y fronteras, Tijuana,

El Colegio de la Frontera Norte / Asociación Latinoamericana de Sociología / El Colegio de México, pp. 425-451.

\section{Notas de pie de página:}

Se utilizarán para hacer indicaciones complementarias, aclaraciones o ampliación de una explicación. La nota de pie de página en Times New Roman, 10 puntos.

\section{Tipología de imágenes dentro del texto:}

Cuadro

Gráfica

Diagrama

Mapa

Figura

Todas las imágenes deben ser numeradas y mencionadas dentro del texto. A toda imagen debe incluirse la fuente.

Las indicaciones de la imagen: tipo y número de imagen, título de imagen y fuente se escriben en 10 puntos. En el texto poner como imagen los mapas, figuras, gráficas y diagramas -con el ánimo de no perder el formato realizado por el autor.

\section{Ecuaciones y fórmulas:}

Si se utilizan ecuaciones o fórmulas deberá utilizarse el editor de ecuaciones de Word y numerarse.

\section{Envío de trabajos}

Los trabajos deben ser enviados a la dirección de correo: lgtz@uacj.mx. Con el Dr. Luis Enrique Gutiérrez Casas, editor de esta publicación.

La aceptación de cada colaboración dependerá de la evaluación de dos dictaminadores especialistas en la materia que se conservarán en el anonimato, al igual que el autor (autores) para efectos de la misma. 


\section{For General Document:}

Font type: Times New Roman.

Size: font size 11.

Paragraph: 1.5 line spacing.

Titles and subtitles: Main text font size 11. Titles font size 12 (Bold). Subtitles font size 11.

Each title and subtitle should be numbered in the following order: 1, 1.1, 2, 2.1, 2.2..

The maximum length of the workbooks will be 40 pages.

The first time an abbreviation is used in the text will be specified first complete equivalence and then stands.

\section{Front cover:}

Title:

Font size 14, centered, Bold.

Author name(s):

Font size 12.

Abstract:

It should include abstract in Spanish and abstract (font size 10), no more than 250 words.

Keywords:

Include three to five keywords, in Spanish and English.

Reference of author:

Institution of affiliation, academic degree and line-developed by research groups and belonging.

\section{Bibliographical appointment system:}

Harvard-APA

Citations in the text should include between parentheses only the author's name, publication date and page number, for example:

(Quilodrán, 2001: 33).

\section{Notation about Bibliography section and Information fonts:}

Should be included at the end of the text. All references must be mentioned in the text or footnotes page.

Each reference starts with the first name or last name, then the name of the author, and then, in parentheses, the year of publication followed by a period. Examples:

Article:

Ros, Jaime (2008). “La desaceleración del crecimiento económico en México desde 1982”, en Trimestre Económico, vol. 75, núm. 299, pp. 537-560. 
Book:

Villarreal, René (2005). Industrialización, competitividad y desequilibrio externo en México. Un enfoque macroindustrial y financiero (1929-2010), México, Fondo de Cultura Económica.

Book chapter:

Castillo, Manuel Ángel (2003). "La política de inmigración en México: un breve recuento", en Manuel Ángel Castillo, Alfredo Lattes y Jorge Santibáñez (coords.), Migración y fronteras, Tijuana, El Colegio de la Frontera Norte / Asociación Latinoamericana de Sociología / El Colegio de México, pp. 425-451.

\section{Footnotes:}

Must be used to make additional indications, clarification or expansion of an explanation. The footnotes must be in Times New Roman, font size 10.

\section{Image typology inside text:}

Picture

Graph

Diagram

Map

Figure

All images must be numbered and mentioned in the text, should include the source image. The indications of the image: type and number of image, image title and source are written in 10 font size. In the text set as image maps, figures, graphs and charts-with the intention of not losing the formatting by the author.

\section{Equations and Formulae:}

When using equations or formulas should be used in Microsoft Word equation editor and numbered.

\section{Paper sending}

Entries must be sent to the email address: 1gtz@uacj.mx. With Dr. Luis Enrique Gutiérrez Casas, editor of this publication.

Acceptance of each collaboration will depend on the evaluation of two examiners skilled in the art to be kept anonymous, like the author(s) for the same purposes. 
Publicación afiliada a la

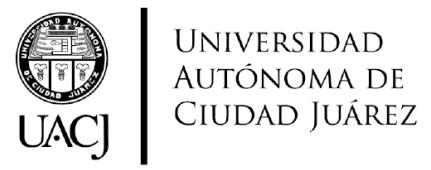

$\begin{aligned} & 0 \begin{array}{l}\text { Red } \\ \text { lberoamericana } \\ \text { de Estudios } \\ \text { del Desarrollo }\end{array} \\ & \text { RIED }\end{aligned}$

Esta obra se editó y terminó de imprimir en

Ciudad Juárez, Chihuahua, México 
